\title{
Experimental Demonstration of a Quantum Receiver Beating the Standard Quantum Limit at Telecom Wavelength
}

Izumi, Shuro; Neergaard-Nielsen, Jonas Schou; Miki, Shigehito; Terai, Hirotaka; Andersen, Ulrik Lund

Published in:

Physical Review Applied

Link to article, DOI:

10.1103/PhysRevApplied.13.054015

Publication date:

2020

Document Version

Publisher's PDF, also known as Version of record

Link back to DTU Orbit

Citation (APA):

Izumi, S., Neergaard-Nielsen, J. S., Miki, S., Terai, H., \& Andersen, U. L. (2020). Experimental Demonstration of a Quantum Receiver Beating the Standard Quantum Limit at Telecom Wavelength. Physical Review Applied, 13(5), [054015]. https://doi.org/10.1103/PhysRevApplied.13.054015

\section{General rights}

Copyright and moral rights for the publications made accessible in the public portal are retained by the authors and/or other copyright owners and it is a condition of accessing publications that users recognise and abide by the legal requirements associated with these rights.

- Users may download and print one copy of any publication from the public portal for the purpose of private study or research.

- You may not further distribute the material or use it for any profit-making activity or commercial gain

- You may freely distribute the URL identifying the publication in the public portal 


\title{
Experimental Demonstration of a Quantum Receiver Beating the Standard Quantum Limit at Telecom Wavelength
}

\author{
Shuro Izumi $\odot,{ }^{1, *}$ Jonas S. Neergaard-Nielsen $\odot,{ }^{1}$ Shigehito Miki, ${ }^{2,3}$ Hirotaka Terai, ${ }^{2}$ and \\ Ulrik L. Andersen ${ }^{1}$ \\ ${ }^{1}$ Center for Macroscopic Quantum States (bigQ), Department of Physics, Technical University of Denmark, \\ Building 307, Fysikvej, 2800 Kongens Lyngby, Denmark \\ ${ }^{2}$ Advanced ICT Research Institute, National Institute of Information and Communications Technology, 588-2, \\ Iwaoka, Nishi-ku, Kobe, Hyogo, 651-2492, Japan \\ ${ }^{3}$ Graduate School of Engineering, Kobe University, 1-1 Rokko-dai cho, Nada-ku, Kobe, 657-0013, Japan
}

(Received 17 January 2020; revised manuscript received 7 March 2020; accepted 13 April 2020; published 7 May 2020)

\begin{abstract}
Discrimination of coherent states beyond the standard quantum limit (SQL) is an important task not only for quantum information processing but also for optical coherent communication. To optimize longdistance optical fiber networks, it is of practical importance to develop a quantum receiver beating the SQL and approaching the quantum bound at telecom wavelength. In this paper, we experimentally demonstrate a receiver beating the conventional SQL at telecom wavelength. Our receiver is composed of a displacement operation, a single-photon counter, and a real-time adaptive-feedback operation. By using a high-performance single-photon detector operating at the telecom wavelength, we achieve a discrimination error beyond the SQL. The demonstration in the telecom band is an enabler for quantum and classical communication beyond the SQL using a coherent state alphabet, and we envision that the technology can be used for long-distance quantum-key distribution, effective quantum-state preparation, and quantum estimation.
\end{abstract}

DOI: 10.1103/PhysRevApplied.13.054015

\section{INTRODUCTION}

Coherent states are inherently nonorthogonal and can therefore not be perfectly discriminated [1]. This nonorthogonality is an attractive feature of coherent states, as it, for example, ensures the security of quantum-key distribution [2,3]. On the other hand, for classical optical coherent communications with a lossy channel such as satellite-to-ground laser communication [4,5] and longdistance optical fiber communication [6,7], the nonorthogonality sets a fundamental limitation on the attainable communication rate and distance. In long-distance coherent fiber communication, optical repeaters (e.g., erbiumdoped fiber amplifiers) are commonly used to amplify weak, attenuated signals. Although these techniques are well established, additional noise in the amplification process limits the communication distance if a cascade of amplifiers is required to transmit a signal [8]. To some extent, this may be remedied by noiseless amplification with phase-sensitive amplifiers $[9,10]$. Another promising and important direction for further improvement of optical coherent communication is designing unconventional receivers detecting the transmitted signal states below the

\footnotetext{
*sizumi@fysik.dtu.dk
}

shot-noise limit [1]. Unlike the strategy relying on amplifiers, which enhances the signal-to-noise ratio for noisy channel communication, the receivers beating the shotnoise limit enables us to attain or closely approach the ultimate performance set by quantum mechanics. Furthermore, the receivers that can directly project an input optical state onto a desired basis are important resources for optical quantum information processing [11]. The efficiency of reading out the information of an encoded signal state is often studied in the context of quantum-state discrimination where the discrimination error of possible candidate signal states is one of the relevant figures of merit.

If one performs direct detection of physical variables that are encoded in coherent states, the discrimination error obtainable is limited by the shot noise. This error probability given by conventional detection techniques is defined as the standard quantum limit (SQL). In quantum mechanics, however, measurements can be mathematically represented as positive-operator-valued measures (POVMs) and the ultimate bound for the discrimination error is obtained by optimizing the POVMs [1]. The minimum discrimination error is called the "Helstrom bound," which can be derived analytically for particular types of states [12] but a physical implementation of 
the optimal measurements is often nontrivial. Receivers beating the SQL, which we call "quantum receivers" hereafter, have been extensively explored both theoretically [13-16] and experimentally [17-21]. For binary-phaseshift-keying (BPSK) signals, performance beyond the SQL is achievable with a simple detection strategy consisting of a displacement operation followed by photon counting [17-20]. Furthermore, the Helstrom bound can be attained by introducing an adaptive-feedback operation that optimizes the displacement operation depending on the outcomes of the photon counting [14,21]. While this feedback receiver was originally proposed for the discrimination of BPSK coherent states, it has been shown that the receiver can implement arbitrary two-dimensional projective measurements [22,23]. Recently, displacementbased photon-detection receivers with or without feedback have been used to realize a single-rail qubit projector that was characterized by quantum-detector tomography [24,25].

In addition to binary-state discrimination, various types of quantum receivers have been developed for quaternaryphase-shift-keying (QPSK) coherent states [26-32]. A significant reduction of the discrimination error beyond the SQL was experimentally observed by optimization of the feedback strategy according to the a posteriori probability for observed events [30]. Moreover, a practical receiver with a photon-number-resolving detector, which is robust against mode-matching imperfection of the displacement and dark-count noise, was analyzed $[29,33]$ and demonstrated [31]. At the telecom wavelengths, however, implementation of quantum receivers that operate beyond the SQL has been particularly challenging mainly due to the low efficiency and high darkcount rates of conventional telecom-based photon counters [34].

In this paper, we experimentally demonstrate an allfiber-based quantum receiver at telecom wavelength that beats the SQL for the discrimination of QPSK states. Our receiver consists of a displacement operation, photon detection, and feedback for updating the displacement. For the photon detection, we use a superconducting-nanowire single-photon detector (SSPD) that shows high performance at the telecom wavelength [35-37]. Our receiver achieves more than $65 \%$ detection efficiency, thereby allowing operation beyond the SQL without compensating for any imperfections. We further investigate the effect of feedback delay in the receiver by developing a mathematical model, and we find that the delay can significantly degrade the performance of the receiver for large signals.

This paper is organized as follows. We first introduce the quantum receiver and derive the theoretically achievable error probability (Sec. II). In Sec. III, we discuss our experimental setup and experimental results, while we provide our conclusions in Sec. IV.

\section{QUANTUM RECEIVER WITH FEEDBACK FOR QPSK SIGNALS}

In this section, we introduce a quantum receiver beating the SQL and approaching the Helstrom bound for the discrimination of the QPSK coherent states.

We define the QPSK coherent states as $\left|\alpha_{m}\right\rangle=$ ||$\alpha\left|e^{(2 m+1) i \pi / 4}\right\rangle$, where $m=0,1,2,3$ and $|\alpha|$ represents the magnitude of the signal state [Fig. 1(a)].

Figure 1(b) depicts a schematic of the receiver. The receiver consists of a displacement operation, a singlephoton detector (SPD) and real-time feedback control of the displacement phase dependent on the counting history of the SPD. The displacement operation can be physically implemented by combining the signal state with a strong reference beam at a beam splitter with nearly unit transmittance. To illustrate the feedback control of the displacement, an incoming signal state with full time width $T$ is virtually divided into $M$ temporal-mode bins. At each step, $i$, of the protocol, a displacement is applied to the $i$ th temporal mode such that one of the candidate states is displaced to the vacuum state. The SPD provides a binary outcome $e_{i} \in\{0$ (off ), 1(on) $\}$ whose POVM is, respectively, represented by $\hat{\Pi}_{0}=e^{-v} \sum_{n=0}^{\infty}(1-\eta)^{n}|n\rangle\langle n|, \hat{\Pi}_{1}=\hat{I}-$ $\hat{\Pi}_{0}$, where $v$ is the dark count noise (counts per state) and $\eta$ is the detection efficiency of the SPD [38]. The probability of getting an outcome $e_{i}$ for a coherent state $|\gamma\rangle$ with a displacement operation $\hat{D}(\beta)=\exp \left(\beta \hat{a}^{\dagger}-\beta^{*} \hat{a}\right)$ is therefore given by

$$
\begin{aligned}
p\left(e_{i} \mid \gamma ; \beta\right)= & \left\langle\gamma\left|\hat{D}^{\dagger}(-\beta) \hat{\Pi}_{e_{i}} \hat{D}(-\beta)\right| \gamma\right\rangle \\
= & \left(1-e_{i}\right) e^{-\nu-\eta|\gamma-\beta|^{2}} \\
& +e_{i}\left(1-e^{-\nu-\eta|\gamma-\beta|^{2}}\right) .
\end{aligned}
$$

(a)

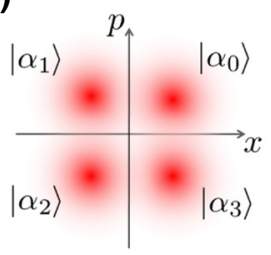

(b)

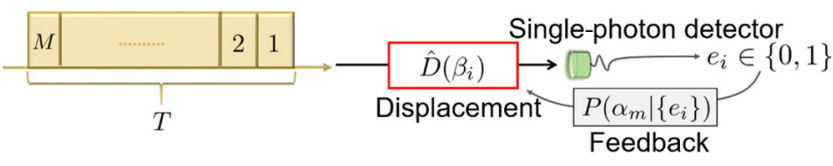

FIG. 1. (a) QPSK coherent states in phase space. (b) A receiver with displacement, photon-detection, and feedback operations. (c) Theoretical performance of the receiver. Solid black, red, blue, and green lines represent the receiver with $M=100, M=$ $10, M=4$, and $M=3$, respective. Dash-dotted and dashed lines represent the standard quantum limit and the Helstrom bound, respectively. 
The a priori belief was that the incoming state was the one that was displaced to the vacuum. If the detector outcome is off, this belief is reinforced and the same displacement is maintained for the next step. If, on the other hand, the outcome is on, this is a strong indication that the incoming state was not displaced to the vacuum. Hence, the displacement phase should (typically) be changed for the next step. On the basis of the full photon-detection history $\left\{e_{r}\right\}$, the candidate state that has the highest $a$ posteriori probability $P\left(\alpha_{m} \mid\left\{e_{i}\right\}\right)$ should now be displaced toward the vacuum state [30].

The a posteriori probability after detection of the $j$ th bin is obtained from

$$
P\left(\alpha_{m} \mid\left\{e_{i}\right\}\right)=\frac{\Pi_{k=1}^{j} p\left(e_{k} \mid \alpha_{m} / \sqrt{M} ; \beta_{k}\right)}{\sum_{l=0}^{3} \Pi_{k=1}^{j} p\left(e_{k} \mid \alpha_{l} / \sqrt{M} ; \beta_{k}\right)},
$$

where we assume that the a priori probabilities of the four signal states are equal and the signal state is equally divided into $M$ temporal modes. The most-likely signal state that maximizes the final a posteriori probability is concluded to be the received state. Thus, the average error probability for the discrimination of the QPSK coherent states using the feedback receiver is represented as

$$
P_{e}=1-\frac{1}{4} \sum_{m=0}^{3} \sum_{\left\{e_{i}\right\} \in O_{m}} P\left(\left\{e_{i}\right\} \mid \alpha_{m}\right),
$$

where the conditional probability $P\left(\left\{e_{i}\right\} \mid \alpha_{m}\right)$ represents the probability of having the outcomes $\left\{e_{i}\right\}$ if the actual incoming signal is $\left|\alpha_{m}\right\rangle$ and $O_{m}$ is the set of outcomes for which it was concluded that the state $\left|\alpha_{m}\right\rangle$ was received. An analytical expression for Eq. (3) was obtained for the ideal case [28]. On the other hand, finding a solution for the general case with experimental imperfections is not straightforward, and we therefore evaluate the error probability with imperfections by simulating the model.

The achievable error probability with the receiver in the ideal condition is shown in Fig. 1(c). The ultimate bound for the discrimination of the QPSK states is given by the Helstrom bound [1,28]. The SQL is the error probability that can be attained by the conventional heterodyne receiver measuring both quadratures, whose error probability is calculated to be

$$
P_{e}^{\mathrm{SQL}}=1-\frac{1}{4}[1+\operatorname{erf}(|\alpha| / \sqrt{2})]^{2},
$$

where erf $x$ is the error function defined as $\operatorname{erf} x=$ $2 / \sqrt{\pi} \int_{0}^{x} e^{-t^{2}} d t$. Under the ideal condition, already the minimum number of feedback steps $M=3$ beats the SQL. Moreover, if the number of feedback steps is increased up to, for example, $M=10$, the feedback measurement closely approaches the Helstrom bound. Although the analytical form for the asymptotic limit of the number of feedback steps $M \rightarrow \infty$ is not straightforward, the reduction of the error probability saturates for large numbers of steps. In contrast to the BPSK case, there is a small gap between the Helstrom bound and the feedback measurement based on photon detection [28].

\section{EXPERIMENT}

Figure 2 illustrates our experimental setup. The beam from a continuous-wave, fiber-coupled laser at $1550 \mathrm{~nm}$ is split into two paths, one for preparation of the signal states and one for the reference field for the displacement operation. We prepare the QPSK signal states using a variable attenuator and a phase shifter that consists of a piezoelectric transducer embedded in a circular mount with an optical fiber looped around to control the intensity and the phase of the signal state, respectively. The displacement operation is physically implemented with use of a 99:1 fiber coupler, where the signal state interferes with the reference field for the displacement operation. The amplitude of the displacement is controlled by an intensity modulator (operating with a constant voltage) that is calibrated to displace the signal states to the vacuum state by minimizing the count rate obtained with a SSPD $[35,36]$. An interference visibility of $99.6 \%$ can be achieved by manual adjustment of the polarization. We switch the laser intensity between high and low using an optical switch to repeatedly and sequentially implement phase stabilization and measurement. For the phase-stabilization period,

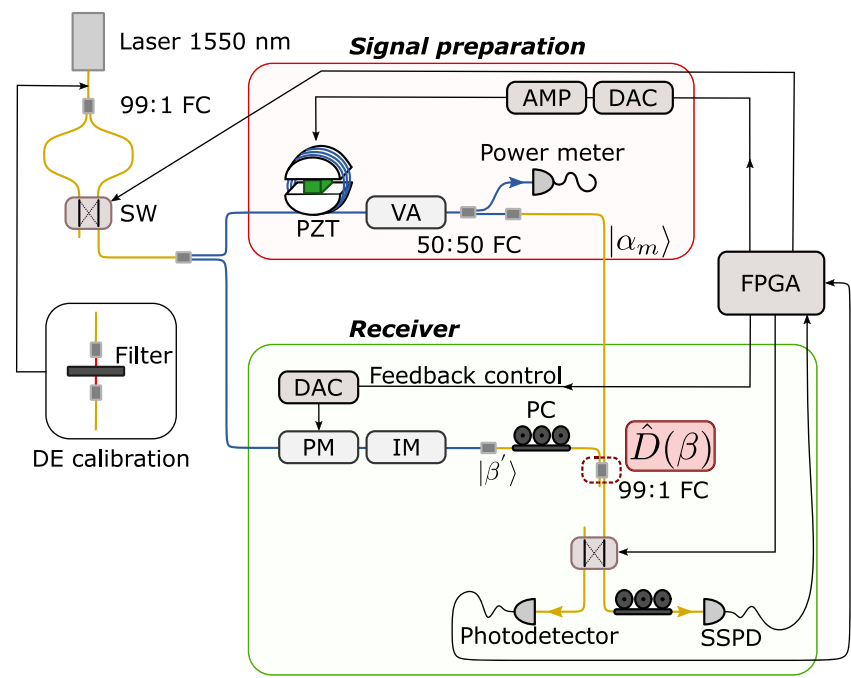

FIG. 2. Experimental setup. Blue and yellow lines represent polarization-maintaining fiber and single-mode fiber, respectively. AMP, amplifier; DE, detection efficiency, FC, fiber coupler; IM, intensity modulator; PC, polarization controller; $\mathrm{PM}$, phase modulator; PZT, piezoelectric transducer; SW, switch; VA, variable attenuator. 
the relative phase between the signal and the reference is set to one of the four phase conditions $(2 m+1) \pi / 4$ $(m=0,1,2,3)$ by our measuring the laser intensity after the 99:1 fiber coupler on a conventional photodetector and feeding back an error signal obtained from the photodetector to the phase shifter. For the measurement, the displaced signal is detected by the SSPD. In our experiment, instead of randomly preparing the QPSK coherent states, we prepare 500 identical signal states after releasing the phase stabilization and do the procedure 20 times for each state. A field-programmable gate array (FPGA) counts the electrical signal from the SSPD and rapidly changes the voltages applied to the phase modulator for the reference field dependent on the counting history, which corresponds to the feedback control of the phase of the displacement operation. The feedback bandwidth of our receiver is about $1 \mathrm{MHz}$, and is mainly limited by the speed of the digital-to-analog converter (DAC) use for the experiment. Since the full time width of the signal state is defined to be $T=200 \mu \mathrm{s}$, the delay of the feedback is not negligible for our experiment and hence we discard the counts observed in the time interval $\Delta t=1.1 \mu$ s between each time bin.

Imperfect transmittance of optical components as well as the nonunit detection efficiency of the SSPD are the main sources of loss in our experiment. The total transmittance from the 99:1 fiber coupler to the fiber immediately before the SSPD $\eta_{T}$ is measured to be approximately $90 \%$ (which is achieved by our splicing the fibers) and the detection efficiency of the SSPD $\eta_{\mathrm{SSPD}}$ can reach approximately $73 \%$ with a dark-count rate of $45 \mathrm{~Hz}$. Therefore, our receiver is expected to have a total system efficiency $\eta_{\mathrm{SE}}=$ $\eta_{T} \times \eta_{\mathrm{SSPD}}$ of about $65 \%$. For characterization of the total system efficiency, we insert free-space filters attenuating the laser power down to a few-hundred-thousand photons, which can be measured by the SSPD without saturation. The laser intensity in the signal-state-preparation path is split into two paths with use of a 50:50 fiber coupler, where the laser power in one path is measured by a power meter to estimate the laser power $I_{\text {in }}$ in the other path guided to the receiver. After insertion of a cascade of calibrated optical filters at the beginning of the setup, the attenuated laser power passing through optical components of the receiver is detected by the SSPD. Since we can estimate the photon rate from the laser power $I_{\text {in }}$ and the attenuation caused by the inserted optical filters, the total system efficiency is obtained by our comparing the photon rate and the observed count rate. The total system efficiency can be evaluated with, at most, $1.5 \%$ uncertainty, including the finite precision of calibrating the filters and the splitting ratio of the 50:50 fiber coupler, the systematic error due to the laser-power fluctuation, and the filter position. As the detection efficiency of the SSPD depends on the polarization of the detected light, the polarization is carefully controlled to optimize the efficiency.

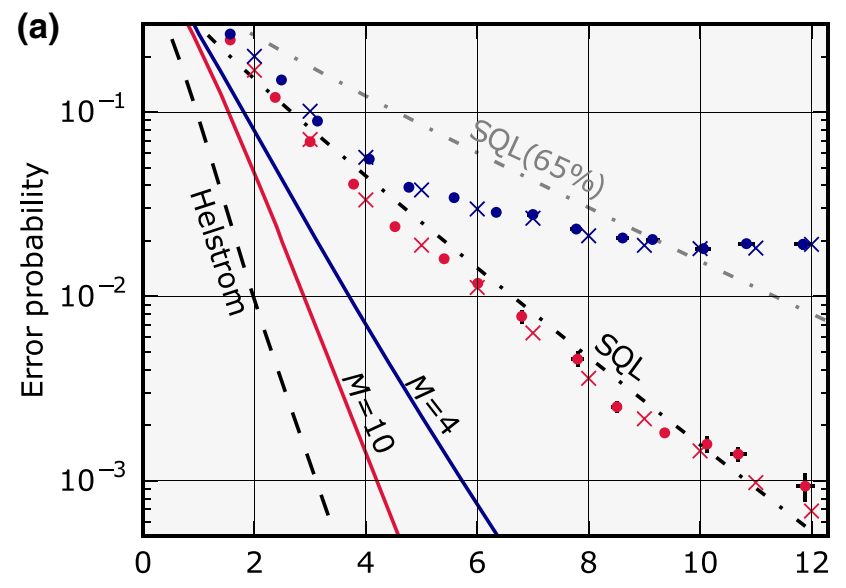

(b)
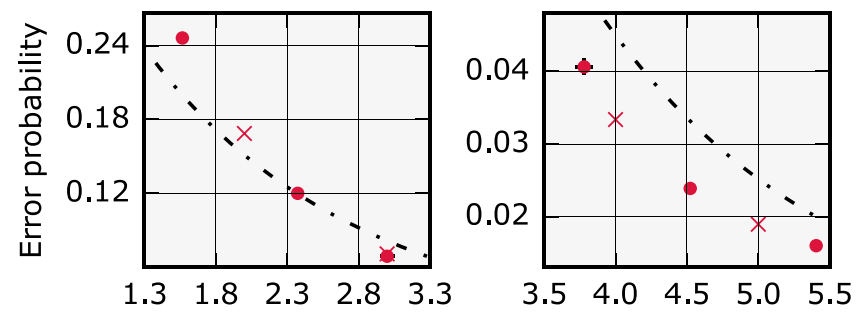

Mean photon number $|a|^{2}$

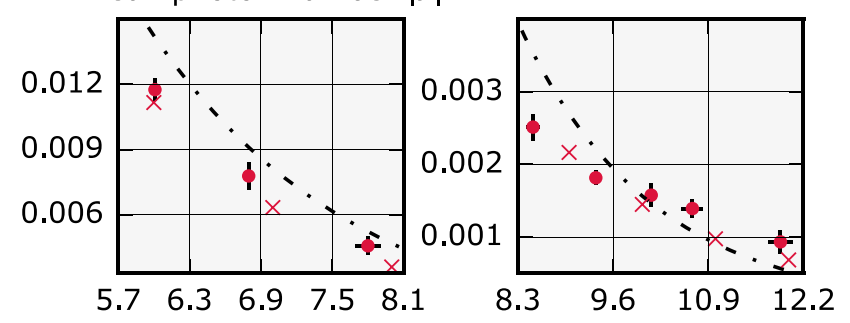

FIG. 3. (a) Error probability for the QPSK discrimination as a function of the signal mean photon number. Red and blue points are experimentally obtained values for $M=10$ and $M=$ 4 , respectively, and the corresponding theoretical predictions are shown by crosses. Red and blue lines represent the performance of the feedback receiver with $M=10$ and $M=4$, respectively, in the ideal condition. Dashed, thick dash-dotted, and thin dashdotted lines represent the Helstrom bound, the SQL and the SQL with total detection efficiency of $65 \%$, respectively. (b) The same data as in (a) but on a linear scale.

The experimentally obtained performance of our receiver is depicted in Figs. 3(a) and 3(b). Blue and red dots represent the experimentally obtained error probability for $M=4$ and $M=10$, respectively. The means and the error bars of the error probability are calculated from five independent procedures. The signal mean photon number $|\alpha|^{2}$ is estimated by our rescaling the attenuated-signal mean photon number $\eta_{\mathrm{SE}}|\alpha|^{2}$ directly observed by the FPGA. The error bar of the signal mean photon number originates from the finite precision of the estimation of the total system efficiency $\eta_{\mathrm{SE}}$. The inconsistent size of the error bars on the signal mean photon 
number is due to the variation of the uncertainty of the total system efficiency associated with its characterization process. We measure the attenuated-signal mean photon number before and after the measurement to ensure that the signal power is stable during the data acquisition, and the difference of the attenuated-signal mean photon numbers is typically less than $1 \%$ of the attenuated-signal power and $3 \%$ at most. As a conservative estimate of the mean photon number, we choose the largest of the two measurements for the plots in Figs. 3(a) and 3(b). The theoretical values shown by crosses are evaluated by Monte Carlo simulations under the experimental conditions, including the total system efficiency $\eta_{\mathrm{SE}}=65 \%$, the visibility $\xi=$ $99.6 \%$, the dark-count noise $v=9.1 \times 10^{-3}$ counts per state, and a loss due to feedback-delay compensation of $4.95 \%$ for $M=10$ and $1.65 \%$ for $M=4$. Dashed, thick dash-dotted, and thin dash-dotted lines represent the Helstrom bound, the SQL and the SQL with total system efficiency $65 \%$. The small deviation of the experimental results from the theoretical values is mainly due to variation of the visibility condition of the displacement. For $M=4$, the saturation of the error probability originates from the imperfection of the visibility and the dark-count noise, which increase the probability of erroneously detecting the photon irrespective of the signal phase condition [29,31]. The effect of these phase-insensitive counts is suppressed by our performing a large number of feedback steps with the feedback strategy based on the a posteriori probability, appropriately taking into account these imperfections. Although the performance of the receiver is far from ideal due to the finite detection efficiency and the visibility of the displacement operations, our receiver with $M=10$ beats the SQL and shows very good agreement with the theoretical values.

We further investigate the possible performance of our receiver with an imaginary SPD assumed to have higher detection efficiencies. In Fig. 4, we plot the expected performances of our receiver with a SPD whose efficiency $\eta_{\text {SPD }}$ is assumed to be $100 \%$ (blue), $90 \%$ (purple), $80 \%$ (green), and $73 \%$ (red), corresponding to our experimental conditions. The signal mean photon number is obtained by our rescaling the attenuated-signal mean photon number $\eta_{T} \eta_{\mathrm{SSPD}}|\alpha|^{2}$, which is directly observed in our experiment, with fixed $\eta_{T}=90 \%$ and variable $\eta_{\mathrm{SPD}}$. Since the maximum detection efficiency of single-photon detectors at telecom wavelength ever reported is above 90\% [37,39], our receiver can potentially provide a significant improvement over the SQL by combining with such SPDs. The lowest requirement for the detection efficiency of the SPD to overcome the SQL with our system is estimated to be $65 \%$, corresponding to total system efficiency of $58.5 \%$.

Apart from the imperfections related to the photon detection, perhaps the most-relevant practical imperfection of the feedback receiver is the limited bandwidth or delay of the feedback operation. During the transition from

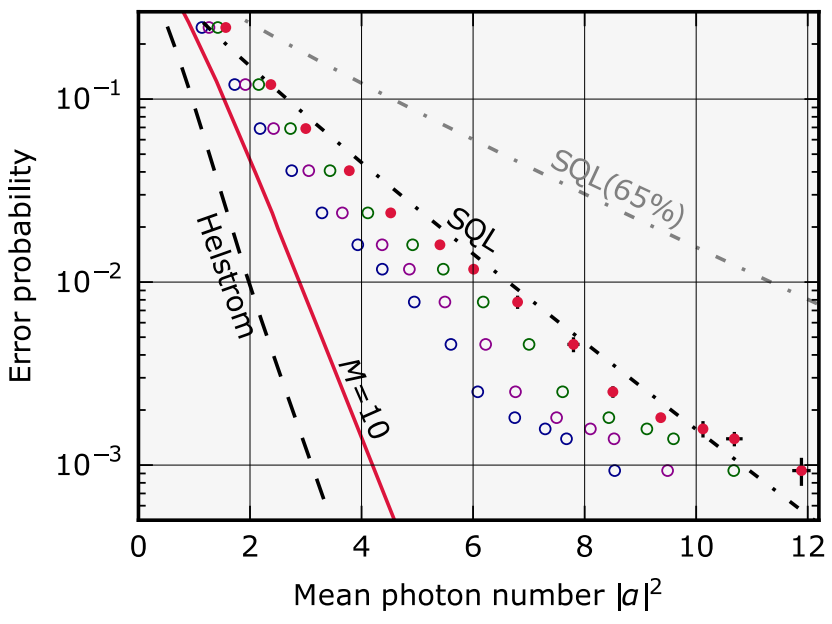

FIG. 4. Expected error probabilities for various detection efficiencies of the SPD. Blue, purple, green, and red points represent the expected error probabilities obtainable with the SPD with detection efficiencies of $100 \%, 90 \%, 80 \%$, and $73 \%$, respectively.

one displacement phase to another, the displacement operation is not well defined and spurious counts may occur, negatively affecting the performance of the receiver. To alleviate the delay problem, the counts observed in a short time interval $\Delta t$ between each temporal-mode bin can be discarded, and the discarding time can be considered as additional linear loss. We characterize this effect by setting the discarding time to various values and observing the error probability. We do this for mean photon numbers $|\alpha|^{2}=3.3$ and $|\alpha|^{2}=9.4$ and plot the results as a function of the discarding time $\Delta t$ in Figs. 5(a) and 5(b). The red circles represent the experimentally obtained error probabilities, whose means and error bars are evaluated from five independent procedures. The black circles and crosses are the theoretical values with and without the delay effect, obtained from the model discussed in Appendix A. The simulation procedure is described in Appendix B. For the black circles, the delay is not considered in the probabilitydistribution model to calculate the a posteriori probability for given outcomes, which is how our receiver is experimentally realized. We further reduce the error probability if the delay effect is taken into account in the probabilitydistribution model to calculate the a posteriori probability but we find that the reduction is not drastic, and our setting the discarding time to $1.1 \mu$ s shows a better performance anyway. Since the displacement phase reaches the target condition within $1.0 \mu \mathrm{s}$, the theoretical values with discarding time $\Delta t$ greater than $1.0 \mu \mathrm{s}$ can be simply analyzed from the model with the discarding loss without the delay effect. The black circles therefore coincide with the black crosses. For the simulation, we take into account the previously mentioned imperfections as well as the discarding loss. The degradation of the performance with small $\Delta t$ is 


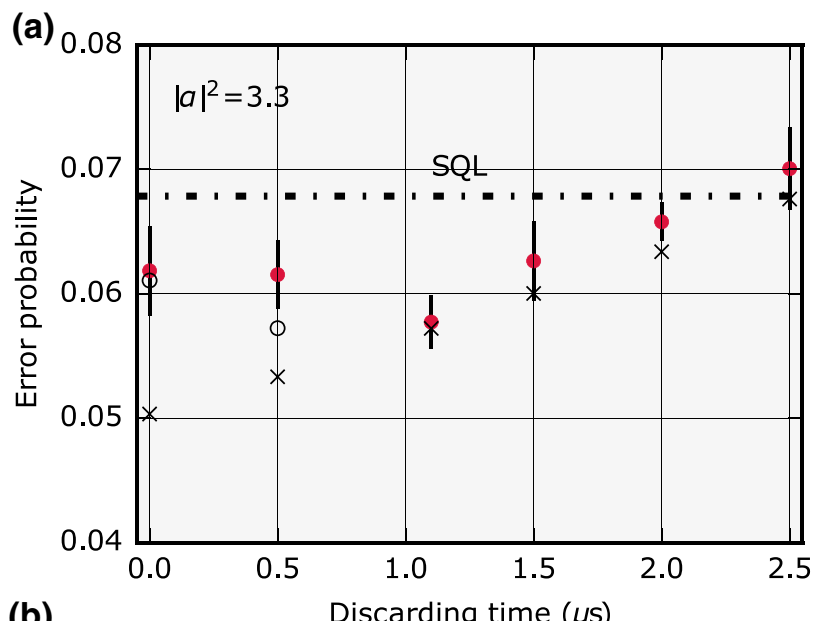

(b)

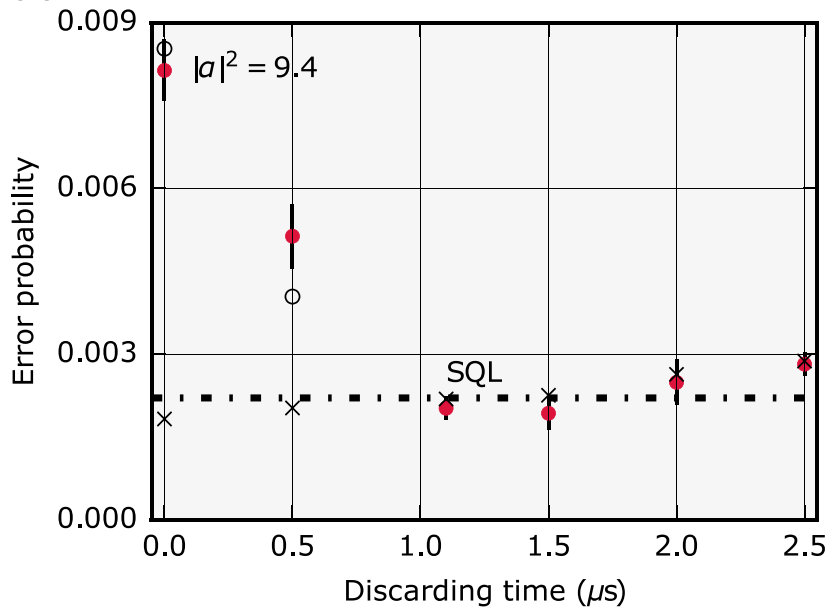

FIG. 5. Error probability for QPSK discrimination with $M=$ 10 as a function of discarding time $\Delta t$ : (a) $|\alpha|^{2}=3.3$; (b) $|\alpha|^{2}=$ 9.4. Red circles represent the experimental results, while black circles and crosses represent the theoretical values with and without the delay effect, respectively. Special treatment of the delay effect is no longer necessary if the discarding time $\Delta t>1.0 \mu \mathrm{s}$ and the black circles are coincident with the black crosses. The dash-dotted line represents the ideal SQL.

due to the delay of the feedback operations, and the error probability can be reduced by our discarding the counts observed in $\Delta t$ but becomes higher as $\Delta t$ increases since the discarding loss increases. Each data point is evaluated from independent experimental procedures and therefore the experimental conditions, such as the mean photon number and the visibility of the displacement, are slightly different in each discarding-time condition. This explains the disagreement between the experimental values and the theoretical values, which are calculated under the same parameter conditions. For small mean photon number, the delay effect is not as critical as for large mean photon number because the probability of detecting photons is low and the phase of the displacement operation is rarely changed.

\section{CONCLUSIONS}

We experimentally realize a quantum receiver consisting of a displacement operation, a single-photon detector, and feedback operation. Our receiver uses a highperformance photon counter and achieves discrimination of QPSK signals with an error probability beating the SQL at telecom wavelength. While the total system efficiency of our receiver is limited to $65 \%$ mainly because of the finite detection efficiency of the photon counter, our system could provide a substantial gain over the SQL if stateof-the-art photon counters showing higher performance at telecom wavelength were installed [37,39]. We further investigate the performance of the feedback measurement with finite bandwidth of the feedback. Our delay analysis shows that the feedback delay can drastically degrade the performance for large signal mean photon numbers, where the displacement operation needs to be changed more frequently.

Since our all-fiber-based telecom quantum receiver is compatible with current optical fiber communication and can be modified to another type of two-dimensional projector such as a single-rail qubit projector [25], we expect that our receiver will provide a practical advantage for not only conventional coherent communication but also quantum-optical information processing [40-42]. An interesting future step is to install our telecom quantum receiver in long-distance fiber communication systems by developing a truly local reference field for the displacement operation $[43,44]$. Such a realistic communication scenario requires high-speed transmission of signal states and therefore the bandwidth of the feedback operation would become a more-critical challenge to be overcome.

Finally, further increase of the feedback bandwidth is, in principle, possible by replacement of the DAC with a faster one. On the other hand, the ultimate feedback bandwidth of our system would be limited by the dead time of the SSPD, which is about $50 \mathrm{~ns}$. One of the promising directions to increase the bandwidth would be to install a multipixel SSPD that can effectively provide a short dead time [45].

\section{ACKNOWLEDGMENTS}

This project was supported by Grant-in-Aid for JSPS Research Fellow, by VILLUM FONDEN via the Young Investigator Programme (Grant No. 10119), and by the Danish National Research Foundation through the Center for Macroscopic Quantum States (Grant No. DNRF142).

\section{APPENDIX A: DELAY ANALYSIS}

Delay of feedback operations may degrade the performance of the receiver. We develop a model of the 


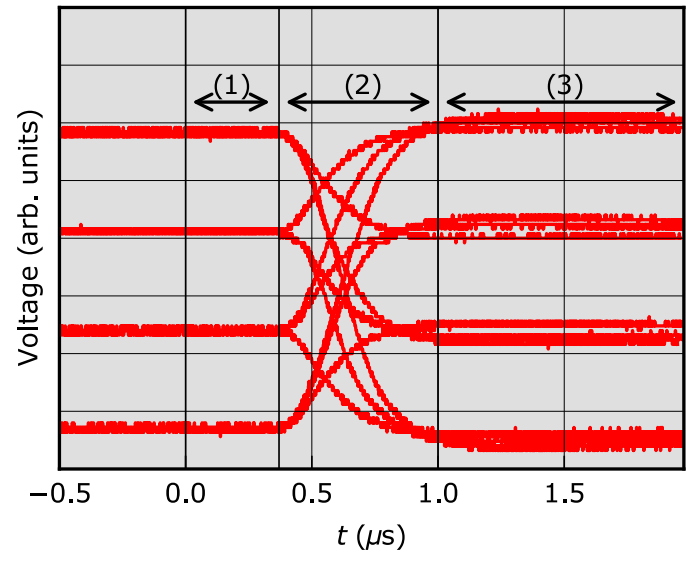

FIG. 6. Electrical signals applied to the phase modulator. The transition between time periods is at $t=0$. The signal remains constant within the temporal region (region 1), changes continuously to the target condition in region 2 , and settles down to the target condition in region).

receiver with finite bandwidth of the feedback operation to analyze the performance in the experimentally relevant condition.

We show the electrical signals applied to the phase modulator in Fig. 6. The $j$ th time bin starts at $t=0$ and the electrical signal remains constant in region 1 , continuously changes to the target condition in region 2 , and settles down to the target condition in region 3 . The delay of the electrical signal is because of the communication speed between the FPGA and the DAC in addition to the finite response time of the DAC. We use a Nexys 4 artix-7 FPGA evaluation board and a Pmod DA3 with 16-bit resolution from Digilent.

To theoretically investigate the performance of the receiver with the delay of the feedback operation, we use spatial-mode analysis where the signal state is spatially divided into $M$ modes and a displacement operation and photon detection are performed on each mode. Suppose that the signal state is equally split and the displacement operation is implemented such that one of the four signal states is displaced to the vacuum state $\hat{D}\left(-\gamma_{m}\right)\left|\gamma_{m}\right\rangle=|0\rangle$, where $\gamma_{m}=\alpha_{m} / \sqrt{M}$. We focus on the second mode to discuss our model for the delay analysis. In the second mode, the signal state is further divided by beam splitters with reflectance (transmittance) $r_{1}^{2}\left(t_{1}^{2}\right)$ and $r_{2}^{2}\left(t_{2}^{2}\right)$ into three steps: (i) the displacement operation remains the same as the previous displacement condition, (ii) the displacement operation changes from the previous condition to the target condition, and (iii) the displacement operation is implemented in the target condition. A schematic of the model is shown in Fig. 7(b). The probability of having the off event in the second mode is given by the product of the off probabilities for all three steps. For steps 1 and 3, the off probability issimply given by

$$
\begin{aligned}
p\left[0 \mid r_{1}\left(\gamma_{m}-\gamma_{0}\right)\right] & =e^{-r_{1}^{2}\left|\gamma_{m}-\gamma_{0}\right|^{2}} \\
& =e^{-2 r_{1}^{2} \gamma^{2}[1-\xi \cos (m \pi / 2)]}, \\
p\left[0 \mid t_{1} t_{2}\left(\gamma_{m}-\gamma_{m_{2}}\right)\right] & =e^{-t_{1}^{2} t_{2}^{2}\left|\gamma_{m}-\gamma_{m_{2}}\right|^{2}} \\
& =e^{-2 t_{1}^{2} t_{2}^{2} \gamma^{2}\left\{1-\xi \cos \left[\left(m-m_{2}\right) \pi / 2\right]\right\}},
\end{aligned}
$$

where $\xi$ is the visibility of the displacement. In step 2, where the displacement operation continuously changes from $\hat{D}\left(-\gamma_{0}\right)$ to $\hat{D}\left(-\gamma_{m_{2}}\right)$, we introduce the following model. The signal state is equally split into $L$ modes and the displacement operation is first implemented such that $m=0$ is displaced to the vacuum state. From the first mode to the $L$ th mode, the phase of the displacement operation is changed with finite step $m_{2} \pi / 2 \times 1 / L$; that is, the displacement operation at the $j$ th mode $(1 \leq j \leq L)$ is given by $\hat{D}\left(-\gamma_{0}^{\prime} e^{i \theta_{j}}\right)$, where $\gamma_{m}^{\prime}=t_{1} r_{2} \gamma_{m} / \sqrt{L}$ and $\theta_{j}=m_{2} \pi / 2 \times$ $(j-1) /(L-1)$. Therefore, the probability of having the off event at the $j$ th mode is given by

$$
\begin{aligned}
p\left(0 \mid \gamma_{m}^{\prime}-\gamma_{0}^{\prime} e^{i \theta_{j}}\right) & =e^{-\left|\gamma_{m}^{\prime}-\gamma_{0}^{\prime} e^{i \theta_{j}}\right|^{2}} \\
& =e^{-2 \gamma^{\prime 2}\left[1-\xi \cos \left(\theta_{j}-m \pi / 2\right)\right]}
\end{aligned}
$$

The off-event probability in step 2 is given by the product of the off probabilities for $L$ modes:

$$
\prod_{j=1}^{L} p\left(0 \mid \gamma_{m}^{\prime}-\gamma_{0}^{\prime} e^{i \theta_{j}}\right)=e^{-\sum_{j=1}^{L} 2 \gamma^{\prime 2}\left[1-\xi \cos \left(m \pi / 2-\theta_{j}\right)\right]}
$$

where

$$
\begin{aligned}
& \sum_{j=1}^{L} 2 \gamma^{\prime 2}\left[1-\xi \cos \left(\frac{m \pi}{2}-\theta_{j}\right)\right] \\
& \quad=2 t_{1}^{2} r_{2}^{2} \gamma^{2}-\sum_{j=1}^{L} \frac{2 t_{1}^{2} r_{2}^{2} \gamma^{2}}{L} \xi \cos \left(\frac{m \pi}{2}-\theta_{j}\right) .
\end{aligned}
$$

To analyze the continuously changing displacement, we consider the limit of infinitely many modes $L \rightarrow \infty$ :

$$
\begin{aligned}
& \lim _{L \rightarrow \infty} \sum_{j=1}^{L} \frac{2 t_{1}^{2} r_{2}^{2} \gamma^{2}}{L} \xi \cos \left(\frac{m \pi}{2}-\theta_{j}\right) \\
& \quad=\frac{4 t_{1}^{2} r_{2}^{2} \gamma^{2}}{m_{2} \pi} \xi\left(\sin \frac{m \pi}{2}-\sin \frac{\left(m-m_{2}\right) \pi}{2}\right) .
\end{aligned}
$$




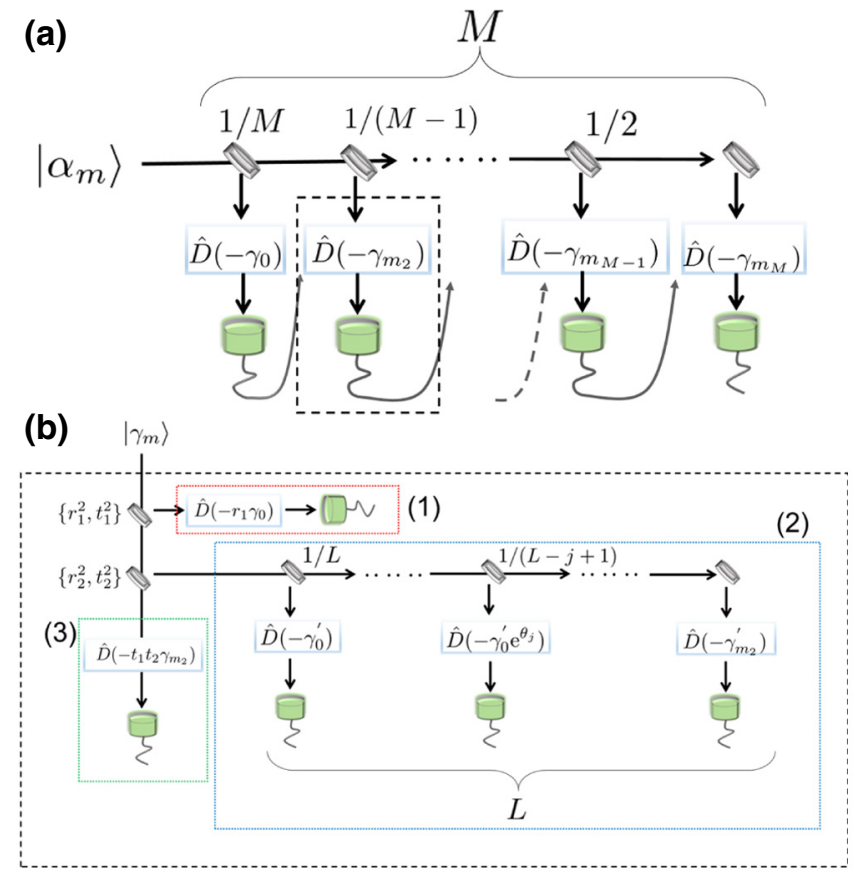

FIG. 7. (a) Feedback measurement with the SPDs in the spatial-mode version. (b) Theoretical model equivalent to the displacement condition with the delay shown in Fig. 6.

The probability of having the off event in step 2 can be analytically obtained as

$$
\begin{aligned}
& \lim _{L \rightarrow \infty} \prod_{j=1}^{L} p\left(0 \mid \gamma_{m}^{\prime}-\gamma_{0}^{\prime} e^{i \theta_{j}}\right) \\
& \quad=e^{-2 t_{1}^{2} r_{2}^{2} \gamma^{2}+\left(4 t_{1}^{2} r_{2}^{2} \gamma^{2} / m_{2} \pi\right) \xi\left\{\sin (m \pi / 2)-\sin \left[\left(m-m_{2}\right) \pi / 2\right]\right\} .}
\end{aligned}
$$

Finally, the probability of having the off event for the second SPD in Fig. 7(a) is obtained from the product of the off-event probabilities for the three detection steps in Fig. 7(b).

Since we define a full time width of the signal state and each time bin as $200 \mu \mathrm{s}$ and $20 \mu \mathrm{s}$ for $M=10$, respectively, from Fig. 6, we find the parameters $r_{1}$ and $r_{2}$ corresponding to our condition to be $r_{1}^{2}=0.37 / 20$ and $r_{2}^{2}=(1.0-0.37) / 20 t_{1}^{2}$. The theoretical values for the performance of the receiver with the feedback delay shown in Fig. 5 are obtained from Monte Carlo simulation based on the model developed above. To simulate the discarding time $\Delta t=0.5 \mu \mathrm{s}$, step 1 is discarded (100\% linear loss), meaning that the outcome from the SPD is always off, and $21 \%$ linear loss $[(0.5-0.37) /(1.0-0.37) \times 100]$ in step 2 is assumed.

In our experiment, we set the discarding time $\Delta t=$ $1.1 \mu \mathrm{s}$ and therefore the associated loss is $1.1 / 200 \times$ $(M-1)$. Figure 8 shows the error probability of the feedback measurement for mean photon number $|\alpha|^{2}=4.0$ as

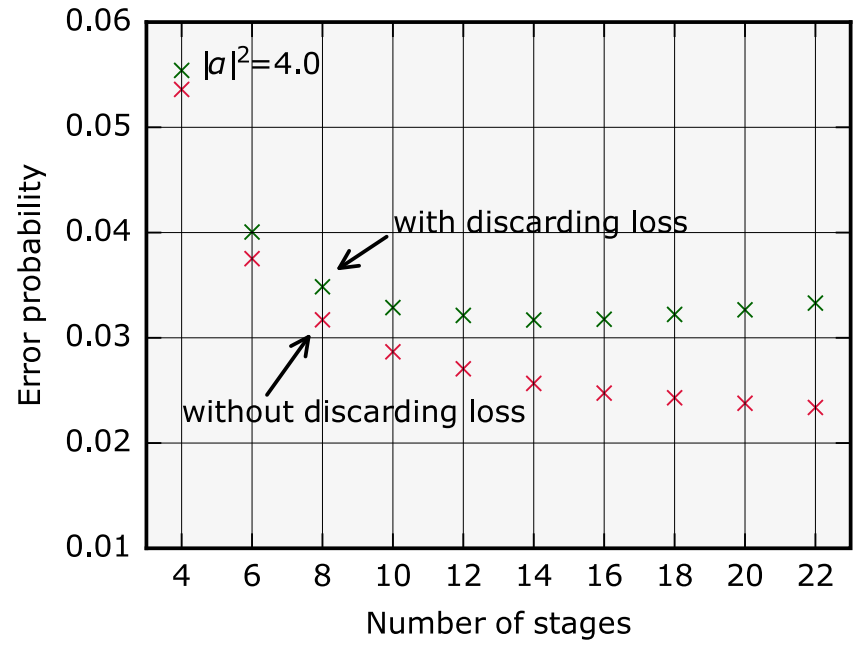

FIG. 8. Error probability for the feedback measurement without the feedback discarding loss (red crosses) and with the discarding loss (green crosses) in the experimental conditions, $\eta=65 \%, \xi=99.6 \%$, and $v=9.1 \times 10^{-3}$ counts per state. The mean photon number of the signal state is set to $|\alpha|^{2}=4.0$.

a function of the number of feedback stages. Red and green crosses represent, respectively, the simulation results for the feedback measurement under the experimental conditions without the discarding loss assuming no delay of the feedback and with the discarding loss due to the delay compensation. For an increasing number of stages, the performance eventually begins to degrade since the discarding loss becomes dominant.

\section{APPENDIX B: SIMULATION}

The theoretically expected values under the experimental conditions are obtained by our performing Monte Carlo simulation. If the displacement magnitude is the same as the magnitude of the signal state, the probability distribution of the photon detection can be described by a Poissonian distribution with mean photon number $\bar{n}=v+$ $2 \eta(1-\xi \cos \theta)|\gamma|^{2}$, where $\theta$ is the relative phase between the signal state and the displacement direction $[31,33,46]$. Hence, the probabilities of having the off event are given by

$$
\begin{aligned}
p\left(0 \mid \gamma_{m} ;-\gamma_{m}\right) & =e^{-\nu-2 \eta(1-\xi)|\gamma|^{2}}, \\
p\left(0 \mid \gamma_{m+\pi / 2} ;-\gamma_{m}\right) & =e^{-\nu-2 \eta|\gamma|^{2}}, \\
p\left(0 \mid \gamma_{m+\pi} ;-\gamma_{m}\right) & =e^{-v-2 \eta(1+\xi)|\gamma|^{2}}, \\
p\left(0 \mid \gamma_{m+3 \pi / 2} ;-\gamma_{m}\right) & =e^{-v-2 \eta|\gamma|^{2}},
\end{aligned}
$$

where $p\left(0 \mid \gamma_{m+\theta} ;-\gamma_{m}\right)$ represents the probability of the off event for the state whose phase is $\theta$ shifted from the target state displaced to the vacuum. For the simulation, we first assume that the signal state $m=0$ is detected by the 
receiver and generate the outcome according to the Poissonian distribution with mean photon number $v+2 \eta(1-$ $\xi)|\gamma|^{2}$. Dependent on the outcome from the SPD, we calculate the a posteriori probabilities $P\left(\alpha_{m} \mid\left\{e_{i}\right\}\right)$ for each state according to Eq. (2) and obtain the most-likely state maximizing the a posteriori probability. In the next step of the stages, the displacement operation is performed such that the most-likely state is displaced to the vacuum state; that is, if the outcome is 0 , corresponding to off, the outcome is generated according to the Poissonian distribution with the same mean photon number and otherwise the outcome is generated according to the Poissonian distribution with the mean photon number dependent on the a posteriori probabilities. By doing the simulation $M$ times and making the final decision on the basis of the a posteriori probability at the last stage, we can conclude whether we successfully identify the incoming signal. We perform a similar procedure assuming that the signal states $m=$ $1,2,3$ are respectively detected by the receiver with the same feedback strategy, and the total error probability is calculated by our summing up the error probabilities for each state divided by 4 (a priori probabilities). We simulate the procedure $10^{6}$ times and obtain the average error probability for the QPSK discrimination with the feedback measurement.

The source code for the theoretical simulation of the strategies discussed in this paper is available at Ref. [47].

[1] C. W. Helstrom, Quantum Detection and Estimation Theory (Academic Press, New York, 1976).

[2] C. H. Bennett and G. Brassard, in Proc. of IEEE International Conference on Computers, Systems, and Signal Processing (IEEE, New York, 1984), p. 175.

[3] N. Gisin, G. Ribordy, W. Tittel, and H. Zbinden, Quantum cryptography, Rev. Mod. Phys. 74, 145 (2002).

[4] V. W. S. Chan, Free-space optical communications, J. Lightwave Technol. 24, 4750 (2006).

[5] S.-K. Liao et al., Satellite-to-ground quantum key distribution, Nature 549, 43 (2017).

[6] K. Kikuchi, Fundamentals of coherent optical fiber communications, J. Lightwave Technol. 34, 157 (2016).

[7] H. Takesue, S. W. Nam, Q. Zhang, R. H. Hadfield, T. Honjo, K. Tamaki, and Y. Yamamoto, Quantum key distribution over a 40-dB channel loss using superconducting singlephoton detectors, Nat. Photonics 1, 343 (2007).

[8] C. M. Caves, Quantum limits on noise in linear amplifiers, Phys. Rev. D 26, 1817 (1982).

[9] Z. Tong, C. Lundström, P. A. Andrekson, C. J. McKinstrie, M. Karlsson, D. J. Blessing, E. Tipsuwannakul, B. J. Puttnam, H. Toda, and L. Grüner-Nielsen, Towards ultrasensitive optical links enabled by low-noise phase-sensitive amplifiers, Nat. Photonics 5, 430 (2011).

[10] M. Asobe, T. Umeki, and O. Tadanaga, Phase sensitive amplifier using periodically poled $\mathrm{LiNbO} 3$ waveguides and their applications, IEICE Trans. Electron. 101-C, 586 (2018).

[11] T. C. Ralph, A. Gilchrist, G. J. Milburn, W. J. Munro, and S. Glancy, Quantum computation with optical coherent states, Phys. Rev. A 68, 042319 (2003).

[12] Y. C. Eldar and G. D. Forney, On quantum detection and the square-root measurement, IEEE Trans. Inf. Theory 47, 858 (2001).

[13] R. S. Kennedy, A near-optimum receiver for the binary coherent state quantum channel, Res. Lab. Electron., MIT, Q. Progr. Rep. 108, 219 (1973).

[14] S. Dolinar, An optimum receiver for the binary coherent state quantum channel, Res. Lab. Electron., MIT, Q. Progr. Rep. 11, 115 (1973).

[15] M. Sasaki and O. Hirota, Optimum decision scheme with a unitary control process for binary quantum-state signals, Phys. Rev. A 54, 2728 (1996).

[16] M. Takeoka and M. Sasaki, Discrimination of the binary coherent signal: Gaussian-operation limit and simple nongaussian near-optimal receivers, Phys. Rev. A 78, 022320 (2008).

[17] C. Wittmann, M. Takeoka, K. N. Cassemiro, M. Sasaki, G. Leuchs, and U. L. Andersen, Demonstration of NearOptimal Discrimination of Optical Coherent States, Phys. Rev. Lett. 101, 210501 (2008).

[18] K. Tsujino, D. Fukuda, G. Fujii, S. Inoue, M. Fujiwara, M. Takeoka, and M. Sasaki, Sub-shot-noise-limit discrimination of on-off keyed coherent signals via a quantum receiver with a superconducting transition edge sensor, Opt. Express 18, 8107 (2010).

[19] K. Tsujino, D. Fukuda, G. Fujii, S. Inoue, M. Fujiwara, M. Takeoka, and M. Sasaki, Quantum Receiver Beyond the Standard Quantum Limit of Coherent Optical Communication, Phys. Rev. Lett. 106, 250503 (2011).

[20] M. T. DiMario and F. E. Becerra, Robust Measurement for the Discrimination of Binary Coherent States, Phys. Rev. Lett. 121, 023603 (2018).

[21] R. L. Cook, P. J. Martin, and J. M. Geremia, Optical coherent state discrimination using a closed-loop quantum measurement, Nature 446, 774 (2007).

[22] M. Takeoka, M. Sasaki, P. van Loock, and N. Lütkenhaus, Implementation of projective measurements with linear optics and continuous photon counting, Phys. Rev. A 71, 022318 (2005).

[23] M. Takeoka, M. Sasaki, and N. Lütkenhaus, Binary Projective Measurement via Linear Optics and Photon Counting, Phys. Rev. Lett. 97, 040502 (2006).

[24] S. Izumi, J. S. Neergaard-Nielsen, and U. L. Andersen, Tomography of a displacement photon counter for discrimination of single-rail optical qubits, J. Phys. B: At. Mol. Opt. Phys. 51, 085502 (2018).

[25] S. Izumi, J. S. Neergaard-Nielsen, and U. L. Andersen, Tomography of a Feedback Measurement with Photon Detection, Phys. Rev. Lett. 124, 070502 (2020).

[26] R. S. Bondurant, Near-quantum optimum receivers for the phase-quadrature coherent-state channel, Opt. Lett. 18, 1896 (1993).

[27] C. R. Müller, M. A. Usuga, C. Wittmann, M. Takeoka, C. Marquardt, U. L. Andersen, and G. Leuchs, Quadrature 
phase shift keying coherent state discrimination via a hybrid receiver, New J. Phys. 14, 083009 (2012).

[28] S. Izumi, M. Takeoka, M. Fujiwara, N. D. Pozza, A. Assalini, K. Ema, and M. Sasaki, Displacement receiver for phase-shift-keyed coherent states, Phys. Rev. A 86, 042328 (2012).

[29] S. Izumi, M. Takeoka, K. Ema, and M. Sasaki, Quantum receivers with squeezing and photon-number-resolving detectors for $M$-ary coherent state discrimination, Phys. Rev. A 87, 042328 (2013).

[30] F. E. Becerra, J. Fan, G. Baumgartner, J. Goldhar, J. T. Kosloski, and A. Migdall, Experimental demonstration of a receiver beating the standard quantum limit for multiple nonorthogonal state discrimination, Nat. Photonics 7, 147 (2013).

[31] F. E. Becerra, J. Fan, and A. Migdall, Photon number resolution enables quantum receiver for realistic coherent optical communications, Nat. Photonics 9, 48 (2015).

[32] A. R. Ferdinand, M. T. DiMario, and F. E. Becerra, Multistate discrimination below the quantum noise limit at the single-photon level, npj Quantum Inf. 3, 43 (2017).

[33] K. Li, Y. Zuo, and B. Zhu, Suppressing the errors due to mode mismatch for $M$-ary PSK quantum receivers using photon-number-resolving detector, IEEE Photonics Technol. Lett. 25, 2182 (2013).

[34] M. L. Shcherbatenko, M. S. Elezov, G. N. Goltsman, and D. V. Sych, Sub-shot-noise-limited fiber-optic quantum receiver, Phys. Rev. A 101, 032306 (2020).

[35] S. Miki, T. Yamashita, H. Terai, and Z. Wang, High performance fiber-coupled $\mathrm{NbTiN}$ superconducting nanowire single photon detectors with Gifford-McMahon cryocooler, Opt. Express 21, 10208 (2013).

[36] T. Yamashita, S. Miki, H. Terai, and Z. Wang, Low-fillingfactor superconducting single photon detector with high system detection efficiency, Opt. Express 21, 27177 (2013).

[37] F. Marsili, V. B. Verma, J. A. Stern, S. Harrington, A. E. Lita, T. Gerrits, I. Vayshenker, B. Baek, M. D. Shaw, R. P.
Mirin, and S. W. Nam, Detecting single infrared photons with 93\% system efficiency, Nat. Photonics 7, 210 (2013).

[38] S. M. Barnett, L. S. Phillips, and D. T. Pegg, Imperfect photodetection as projection onto mixed states, Opt. Commun. 158, 45 (1998).

[39] A. E. Lita, A. J. Miller, and S. W. Nam, Counting nearinfrared single-photons with $95 \%$ efficiency, Opt. Express 16, 3032 (2008).

[40] A. Acín, N. Brunner, N. Gisin, S. Massar, S. Pironio, and V. Scarani, Device-Independent Security of Quantum Cryptography Against Collective Attacks, Phys. Rev. Lett. 98, 230501 (2007).

[41] R. Chaves and J. B. Brask, Feasibility of loophole-free nonlocality tests with a single photon, Phys. Rev. A 84, 062110 (2011).

[42] C. Branciard, E. G. Cavalcanti, S. P. Walborn, V. Scarani, and H. M. Wiseman, One-sided device-independent quantum key distribution: Security, feasibility, and the connection with steering, Phys. Rev. A 85, 010301 (2012).

[43] M. Bina, A. Allevi, M. Bondani, and S. Olivares, Phase-reference monitoring in coherent-state discrimination assisted by a photon-number resolving detector, Sci. Rep. 6, 26025 (2016).

[44] S. Izumi, M. Takeoka, K. Wakui, M. Fujiwara, K. Ema, and M. Sasaki, Optical phase estimation via the coherent state and displaced-photon counting, Phys. Rev. A 94, 033842 (2016).

[45] M. S. Allman, V. B. Verma, M. Stevens, T. Gerrits, R. D. Horansky, A. E. Lita, F. Marsili, A. Beyer, M. D. Shaw, D. Kumor, R. Mirin, and S. W. Nam, A near-infrared 64-pixel superconducting nanowire single photon detector array with integrated multiplexed readout, Appl. Phys. Lett. 106, 192601 (2015).

[46] M. Takeoka, K. Tsujino, and M. Sasaki, Cut-off rate analysis of practical quantum receivers, J. Mod. Opt. 57, 207 (2010).

[47] https://github.com/qpit/QPSK_discrimination.git. 